Société d'histoire de la révolution de 1848 et des

révolutions du XIXe siècle

$61 \mid 2020$

Dans l'intimité de l'exil

\title{
Géraldine BARRON, Edmond Pâris et l'art naval. Des pirogues aux cuirassés, préface de Liliane HILAIRE- PÉREZ
}

Jean-Louis Lenhof

\section{CpenEdition}

Journals

Édition électronique

URL : https://journals.openedition.org/rh19/7275

DOI : $10.4000 /$ rh 19.7275

ISSN : $1777-5329$

Éditeur

La Société de 1848

Édition imprimée

Date de publication : 1 décembre 2020

Pagination : 282-284

ISSN : 1265-1354

\section{Référence électronique}

Jean-Louis Lenhof, « Géraldine BARRON, Edmond Pâris et l'art naval. Des pirogues aux cuirassés, préface de Liliane HILAIRE-PÉREZ », Revue d'histoire du XIXe siècle [En ligne], 61 | 2020, mis en ligne le 20 janvier 2021, consulté le 20 août 2021. URL : http://journals.openedition.org/rh19/7275 ; DOI : https:// doi.org/10.4000/rh19.7275

Ce document a été généré automatiquement le 20 août 2021.

Tous droits réservés 


\title{
Géraldine BARRON, Edmond Pâris et l'art naval. Des pirogues aux cuirassés, préface de Liliane HILAIRE-PÉREZ
}

\author{
Jean-Louis Lenhof
}

\section{RÉFÉRENCE}

Géraldine BARRON, Edmond Pâris et l'art naval. Des pirogues aux cuirassés, préface de Liliane HILAIRE-PÉREZ, Toulouse, Presses Universitaires du Midi, coll. « Histoire et Techniques ", 2019, 261 p., $28 €$.

1 Cet ouvrage est une version remaniée d'une thèse de doctorat qui fut à raison remarquée par les spécialistes lors de sa soutenance en 2015. Géraldine Barron, chartiste, actuellement conservatrice à la bibliothèque de l'Université du Littoral-Côte d'Opale (un haut lieu de la recherche en histoire maritime), a adopté dans sa thèse un point de vue situé à la convergence entre l'histoire navale et l'histoire des techniques de l'époque contemporaine. L'approche, dans les deux domaines considérés, allait dans le sens des renouvellements en cours : d'une part l'attention portée aux personnels et métiers de la marine de guerre, d'autre part l'accent mis sur le rôle des praticiens et des passeurs d'innovation. Le choix de la figure originale que fut l'amiral français Edmond Pâris (1806-1893) s'est avéré pleinement judicieux pour mener une telle démarche croisée. Prenant à bras-le-corps un vaste corpus de sources manuscrites et imprimées, armée d'une excellente maîtrise de la technicité nautique, Géraldine Barron a rendu service à l'avancement de la science. Tout cela transparaît à l'évidence dans l'ouvrage qu'elle vient de livrer au public.

2 L'officier de marine Edmond Pâris a eu une longue carrière, commencée sous la Restauration et terminée, pour sa partie active, au début de la III ${ }^{\mathrm{e}}$ République - carrière brillante, couronnée par son accession au grade de vice-amiral. Chronologiquement, cette carrière a placé l'intéressé au cœur de la « révolution navale» qui a, entre les 
années 1830 et les années 1870, profondément renouvelé les techniques de la marine de guerre et modifié considérablement le travail et la manière de faire des différentes catégories de personnel. C'est ce que rappelle Géraldine Barron en introduction. Mais l'ouvrage met moins l'accent sur les états de service d'Edmond Pâris que sur son itinéraire et ses œuvres de "marin savant». La marine de guerre des XVIII et $\mathrm{XIX}^{\mathrm{e}}$ siècles ne fut pas avare de ce genre d'officier, mais Edmond Pâris s'est surpassé en la matière. Excellent marin, par ailleurs doué en hydrographie et en dessin, cet officier a navigué sur toutes les mers du globe, commandé des bâtiments et combattu pour son pays. Il a été, de plus, un acteur de la transition entre la voile et la vapeur, entre le bois et le cuirassement en fer, entre l'artillerie à âme lisse et celle à âme rayée. Acteur engagé, soucieux de réfléchir à la marche des choses, de prendre du recul et de publier sur tout cela, cela lui valut d'être élu membre de l'Académie des Sciences. En même temps, et justement parce que les temps changeaient, Edmond Pâris a œuvré tout au long de sa vie à une patrimonialisation des techniques nautiques innovantes.

3 C'est ce que montre avec force Géraldine Barron dans cet ouvrage à l'édition soignée, qui n'a pas sacrifié l'appareil scientifique. Prenant du champ par rapport à une optique chronologique platement linéaire, le propos s'articule en quatre parties thématiques qui ont l'intérêt de replacer le cas Pâris dans les enjeux de l'époque et de le connecter à des questions historiographiques de premier ordre. Les deux premières parties dégagent les deux aspects saillants de la carrière de l'amiral Pâris : d'une part, son rôle dans le développement de la marine de guerre à vapeur; d'autre part, son action en ce qui concerne la mémoire de la construction navale. Une troisième partie met en lumière la manière dont l'expertise de Pâris à propos de la marine des temps nouveaux s'est déployée et a été reconnue par ses contemporains. La quatrième partie s'intéresse à l'œuvre écrite de l'amiral, œuvre aussi bien savante que de vulgarisation.

Edmond Pâris a commencé sa carrière à bord de bâtiments en bois, propulsés à la voile seule, mais après la fin de la grande confrontation navale franco-britannique, donc dans ces derniers temps de la marine de guerre non mécanisée, au cours desquels les navires de guerre ont surtout servi à "montrer le pavillon", et à parfaire la connaissance du globe, de ses espaces maritimes et de ses populations. À ce titre, Pâris a eu la chance d'enchaîner trois tours du monde, nullement effectués dans la hâte. Il en a ramené de précieuses informations sur la construction navale chez les peuples extraeuropéens, spécialement ceux de l'aire Pacifique - ce qui lui a permis de s'affirmer dans le domaine des publications savantes dès le début des années 1840 . Ce souci du patrimoine nautique et de sa mémoire, il l'a retrouvé dans ses fonctions à la tête du musée de la Marine, à partir de 1871: loin de considérer ces fonctions comme une sinécure destinée à se reposer d'un intense service actif, Edmond Pâris a insufflé une nouvelle vie au musée en question, ce qui était crucial dans un pays fondamentalement terrien comme la France et qui, en même temps, ne pouvait se passer d'être une puissance maritime.

5 Le cœur de l'ouvrage de Géraldine Barron se trouve néanmoins dans tout ce qui concerne la modernisation de la marine de guerre et particulièrement l'adoption de la vapeur. L'articulation entre invention, innovation et diffusion n'est pas jamais simple, particulièrement dans la marine de guerre. La mécanisation de la propulsion était une technique d'origine "terrienne ", qui avait d'abord été testée, du reste avec succès, dans la marine civile (marchande). Elle eut du mal à s'imposer à bord des bâtiments de l'État, même en France, pays pourtant pionnier en matière de révolution navale. Encore 
jeune, elle n'était techniquement pas facile à concilier avec les fonctions de combat et de projection de forces worldwide des navires de guerre. Les milieux dirigeants de la marine craignaient, en innovant trop rapidement, de fragiliser un outil d'une telle importance géostratégique. D'où l'importance de l'engagement d'officiers influents, reconnus comme marins et militaires de premier ordre, dans la promotion des nouveaux matériels. Géraldine Barron montre de manière convaincante comment Edmond Pâris fut l'un de ces officiers. Intéressé très tôt à la machine à vapeur, au point de perdre un avant-bras en s'approchant trop près d'un engrenage, il a commandé des navires de guerre à vapeur de taille croissante, y compris au combat, durant cette guerre de Crimée qui fut le tournant décisif de la révolution navale. Empiétant sur les compétences des ingénieurs, il a même contribué à perfectionner les engins moteurs.

6 Au reste, Edmond Pâris ne s'est pas contenté de promouvoir la cause de la marine des temps nouveaux dans les milieux autorisés, au sein d'un étroit groupe professionnel et parmi les décideurs politiques. Il a su bâtir tout un réseau et se connecter à un environnement social et intellectuel plus large, savant et au-delà. Il a ainsi participé, de manière d'ailleurs lucide, à une ambiance prométhéenne et progressiste, d'ouverture sur le monde, que l'on retrouve dans l'œuvre d'un Jules Verne - par ailleurs si féru d'aventures maritimes. Géraldine Barron passe en revue, catégorise, quantifie, contextualise et analyse les diverses publications d'Edmond Pâris. Elle explique quel "technologue» il fut, quel pilier de l'édition technique et maritime il est devenu. Edmond Pâris s'est employé à rédiger des manuels pour l'usage des nouvelles techniques, tel le Catéchisme du mécanicien à vapeur (1857). Mais il a aussi fait œuvre de conservateur, de codificateur et de lexicographe comme co-auteur d'un célèbre Dictionnaire de la marine à voile et à vapeur (1848), régulièrement réédité, puis en publiant un Traité des manœuvres à bord des bâtiments à voiles et à vapeur (1866). Avec de telles sommes, il entendait à la fois faire œuvre utile pour les futurs professionnels, offrir à un large public une connaissance rigoureuse des pratiques et métiers de la mer, et embrasser dans un même mouvement l'ancien (en train de disparaître) et le nouveau (en train de naître). Avant que Géraldine Barron ne s'intéresse à lui, l'amiral Pâris restait surtout connu pour son Dictionnaire. Elle a bien fait de ressortir d'un relatif oubli cette grande figure de "passeur", de professionnel de haute technicité capable de réflexion et d'influence intellectuelle. Cette figure, le présent ouvrage la remet pleinement en lumière. 